\title{
Limitaciones al uso de los marcadores tumorales séricos en la práctica oncológica
}

A. Sánchez-Muñoz, A. M. García Tapiador, E. Martínez Ortega, R. Dueñas García, J. Pérez-Regadera Gómez, A. L. Ortega Granados, P. Sánchez-Rovira, A. Lozano Barriuso

\section{Resumen}

Los marcadores tumorales séricos (MTS) son utilizados de manera habitual en la monitorización de la respuesta y en el seguimiento de los pacientes oncológicos tras las diversas terapias administradas.

Presentamos tres casos clínicos con falsas elevaciones de MTS, como son CA 15-3, CA 12-5 y CA 19-9, en pacientes diagnosticados y tratados de carcinoma de mama, carcinoma de ovario y carcinoma colorrectal respectivamente, durante su seguimiento.

A continuación se discuten las limitaciones de su uso en la práctica oncológica como consecuencia de sus problemas de sensibilidad y especificidad, y cual es su utilidad en el pronóstico, supervivencia y calidad de vida de los pacientes.

\section{Palabras clave:}

Marcadores tumorales séricos. Seguimiento. Monitorización de la respuesta al tratamiento.

Oncología, 2005; 28 (9):443-447 


\section{Summary}

Serum tumor markers are often used for the evaluation of the response and follow-up of oncologic patiets after different dispensed therapies.

We report three clinicl cases of false elevation of CA 15-3, CA 12-5 and CA 19-9 serum tumor markers during the follow-up of patients diagnosed and treated for breast cancer, ovarian cancer and colorectal cancer respectively.

The limitations to their use in oncologic practice are discussed in relation with sensitivity and specificity problems, as well as their usefulness in the prognosis, survival and quality of life considerations of the patients.

Key words: Serum tumor markers. Surveillance. Treatment monitoring.

\section{Introducción}

El uso de los MTS ocurre de manera habitual en la monitorización de la respuesta y en el seguimiento de los pacientes oncológicos tras las terapias administradas. Sin embargo, las limitaciones en cuanto a la sensibilidad y especificidad de estos marcadores plantea en ocasiones problemas en la interpretación de sus resultados por parte del oncólogo médico. En los siguientes tres casos clínicos describimos situaciones de falsas elevaciones de MTS, como son CA 15-3, CA 12-5 y CA 19-9, en pacientes diagnosticados y tratados de carcinoma de mama, carcinoma de ovario y carcinoma colorrectal respectivamente, durante su seguimiento.

\section{Casos clínicos}

\section{Caso 1}

Mujer de 44 años de edad sin antecedentes personales de interés. En agosto de 1999 fue diagnosticada de un carcinoma de mama ductal infiltrante pobremente diferenciado pT3N1M0 con receptores hormonales negativos. Fue intervenida mediante mastectomía radical con vaciamiento axilar. El estudio de extensión fue negativo. Una tomografía axial computerizada (TAC) objetivó la presencia de una lesión redondeada única en lóbulo hepático derecho de $4 \mathrm{~cm}$ compatible con hemangioma hepático. El marcador tumoral CA 15-3 fue de $20 \mathrm{U} / \mathrm{ml}(0-25)$.
La paciente recibió tratamiento quimioterápico adyuvante con ciclofosfamida, epirubicina y 5-fluorouracilo, y posterior radioterapia local. En las siguientes revisiones los valores de CA 15-3 fueron ascendiendo de modo progresivo hasta un valor máximo de $55 \mathrm{U} / \mathrm{ml}$ en febrero de 2004. Los estudios radiológicos no detectaron recidiva tumoral, permaneciendo la citada imagen hepática única sin cambio alguno. La paciente presentaba un cuadro de astenia y aumento de peso en los últimos meses. La analítica reveló una hipercolesterolemia de $280 \mathrm{mg} / \mathrm{dl}$ (150-220) y un hipotiroidismo clínico con TSH 43 $\mathrm{U} / \mathrm{mL}(0.27-4.2)$ y $\mathrm{T} 4$ libre de $0.4 \mathrm{ng} / 100 \mathrm{~mL}(0.93-$ 1.7). Se pautó tratamiento sustitutivo mediante levotiroxina con normalización de la función tiroidea. En los meses siguientes los valores de CA 15-3 descendieron siendo su último valor de $26 \mathrm{U} / \mathrm{ml}$ en abril de 2005. En el momento actual la paciente sigue revisiones periódicas sin evidencia de recidiva tumoral.

\section{Caso 2}

Mujer de 67 años de edad con el antecedente de una hepatopatía crónica por virus de la hepatitis C. En julio de 2000 fue sometida a cirugía por un cuadro abdominal agudo. Se diagnosticó de un carcinoma de ovario seroso-papilar bien diferenciado estadio IC. Posteriormente se administraron 6 ciclos de quimioterapia adyuvante con paclitaxel y carboplatino. El valor del marcador tumoral CA 12-5 era de 35 U/ml (0-30). La bioquímica sanguínea fue nor- 
mal excepto GPT de 62 U/L (10-47) y gamma GT de 74 U/L (7-32). Desde entonces la paciente ha seguido revisiones periódicas sin evidencia clínica o radiológica de recidiva tumoral. Los valores del CA $12-5$ han oscilado entre 32 y $54 \mathrm{U} / \mathrm{ml}$. El resto de la analítica no ha presentado alteraciones excepto leve o moderado aumento de las enzimas hepáticas. En su última revisión en mayo de 2005 sigue sin evidencia de enfermedad oncológica.

\section{Caso 3}

Varón de 73 años con antecedentes de litiasis biliar, fue diagnosticado en junio de 1999 de un adenocarcinoma de recto bien diferenciado a $4 \mathrm{~cm}$ del margen anal estadio B2. Se trató mediante amputación abdomino-perineal y posterior quimioterapia con 5-fluorouracilo en infusión continua y radioterapia concurrente. El estudio de extensión no encontró enfermedad a distancia. Los valores iniciales tras la cirugía de los marcadores tumorales CEA y CA 199 fueron de $1 \mathrm{ng} / \mathrm{ml}(0-5)$ y $5.27 \mathrm{U} / \mathrm{ml}(0-37)$ respectivamente. El resto de la bioquímica fue normal. A los 6 meses de finalizar la quimioradioterapia concurrente el valor de CA 19-9 era $73 \mathrm{U} / \mathrm{ml}$. En las sucesivas revisiones los valores de CA 19-9 han oscilado de manera ondulante entre 47 y $489 \mathrm{U} / \mathrm{ml}$, en las diversas revisiones realizadas. En repetidas ocasiones se han realizado estudios radiológicos mediante TAC, RNM en las que sólo se ha visualizado una imagen de dilatación del uréter izquierdo en todo su trayecto hasta la altura de la pelvis compatible con una obstrucción crónica que no se ha modificado en los controles evolutivos, así como hasta tres tomografías por emisión de positrones (PET) y varias colonoscopias por el orificio de la colostomía sin evidenciarse foco de recurrencia de enfermedad. En su última revisión efectuada en junio de 2005 el paciente estaba completamente asintomático y sin evidencia de enfermedad tumoral, con un valor de CA 19-9 de $78 \mathrm{U} / \mathrm{ml}$.

\section{Discusión}

En la práctica oncológica actual cada vez se ha extendido más el uso de marcadores tumorales que son detectados en el suero o fluidos biológicos de los pacientes, y permiten ser cuantificados debido a su capacidad antigénica mediante técnicas de inmunohistoquímica con anticuerpos monoclonales.

Con ello pretendemos conseguir un beneficio clínico para los pacientes mediante un diagnóstico precoz del cáncer o de su recidiva y la instauración de un tratamiento inmediato que aumente su supervivencia, la posibilidad de curación o proporcione una mejoría de su calidad de vida. En otras ocasiones buscamos predecir la respuesta de la enfermedad a los diversos tratamientos oncológicos o una información sobre el pronóstico de los pacientes. Sin embargo, la validez de los MTS está limitada en su aplicación clínica, tanto en la monitorización de la respuesta al tratamiento y sobre todo en el seguimiento de los pacientes, debido a la aparición de valores falsos positivos (elevación del marcador en ausencia de evento clínico que se pretende evidenciar) o falsos negativos (ausencia de elevación del marcador en presencia del evento clínico) ${ }^{1}$.

El CA $12-5$ es una glicoproteína de alto peso molecular (mucina) que se sintetiza en los epitelios derivados de la cavidad celómica. Se encuentra en estructuras variadas como pleura, peritoneo, pericardio, miocardio, trompa de Falopio, endometrio, endocérvix y fondo vaginal. Por tanto, puede ser sintetizada tanto por células normales como malignas. Se emplea como marcador en la evolución del cáncer de ovario tanto en su tratamiento como en su seguimiento. Este marcador puede estar elevado en otras neoplasias como adenocarcinomas (mama, pulmón, de origen digestivo), linfomas, leiomiosarcomas o melanomas, así como en procesos patológicos benignos como patología ginecológica (tumores benignos, salpingitis agudas o crónicas, abscesos tuboováricos, endometriosis), hepatopatías (granulomatosis hepática, hepatitis crónica alcohólica o vírica), pancreatitis, insuficiencia renal, derrame pericárdico o pleural, sarcoidosis, tuberculosis peritoneal, colagenosis, procedimientos quirúrgicos o que causan una rotura traumática del peritoneo, como la diálisis peritoneal ${ }^{2,3}$.

Una utilidad potencial de CA $12-5$ es el valor de este marcador como factor pronóstico de supervivencia en el carcinoma epitelial de ovario ${ }^{4,5}$. Cuando el CA 12-5 no desciende a valores normales tras los tres primeros ciclos de quimioterapia, las posibilidades de alcanzar una respuesta patológica completa son remotas. La elevación de CA 12-5 en una paciente tratada previamente por cáncer de ovario, 
se acompaña o precede a la recidiva clínica en un 90\% de los casos, incluso meses antes de su detección, si bien no está claro si ello contribuye a aumentar la supervivencia de las enfermas en comparación a la actitud de esperar e iniciar tratamiento tras verificar la recurrencia clínica o por métodos radiológicos.

El CA 15-3 es una glicoproteína de alto peso molecular (mucina) usada principalmente en el diagnóstico, seguimiento y evaluación de la respuesta en el cáncer de mama. CA 15-3 también puede aparecer elevada en otros carcinomas (ovario, pulmón, páncreas, hígado), y en patologías no oncológicas, como tumores benignos de ovario, enfermedades benignas de la mama, hepatopatía crónica o cirrosis hepática, sarcoidosis, tuberculosis, lupus eritematoso sistémico o disfunción tiroidea ${ }^{2,6,7}$.

El CA 19-9 es el marcador tumoral gastrointestinal más ampliamente estudiado después del CEA (antígeno carcinoembrionario). El análisis de CA 19-9 detecta una mucina que contiene el sialato de Lewis, un epítope pentasacárido, fucopentosa II. Es el mejor marcador disponible para el adenocarcinoma de páncreas si bien parece aportar poca información extra a la del CEA en la monitorización de pacientes con carcinoma colorrectal. El CA 19-9 también puede estar elevado en otros adenocarcinomas como el gástrico, pulmonar o hepatobiliar, y en patología no tumoral de la vía biliar intra y extrahepática o pancreática ${ }^{2}$.

En este artículo presentamos tres casos clínicos, ejemplos de falsas elevaciones de MTS en el seguimiento de pacientes oncológicos. En dos de ellos hemos identificado sus causas probables como son la hepatopatía crónica por VHC y el hipotiroidismo, ambas descritas en la literatura. Los tres pacientes fueron investigados mediante exploraciones complementarias, en ningún momento se administró algún tipo de terapia oncológica y tras un seguimiento prolongado durante años no se ha evidenciado una recurrencia tumoral. Por tanto, es razonable afirmar que tales elevaciones no son consecuencia de una recurrencia del tumor.

En el año 2000 la Sociedad Americana de Oncología Clínica (ASCO) publicó una guía clínica actualizada sobre las recomendaciones del uso de marcadores tumorales en el cáncer de mama y colorrectal. El panel de miembros consideró que los datos eran insuficientes para recomendar el uso de CA 19-
9, CEA y CA 15-3 para el screening, diagnóstico, estadiaje o seguimiento de las pacientes tras su tratamiento $^{8}$. De igual modo no se acepta el uso de CA12-5 como test de screening fuera de ensayo clínico para el carcinoma de ovario ${ }^{9}$, y como ya se ha comentado, su determinación durante el seguimiento de las pacientes puede adelantar en meses el diagnóstico de la recurrencia, pero no está demostrado si ello contribuye a aumentar su supervivencia ${ }^{10}$.

En nuestra opinión, el empleo de los MTS en el seguimiento de los pacientes tras su tratamiento oncológico, plantea problemas de sensibilidad y sobre todo de especificidad no habiéndose demostrado su influencia favorable en la supervivencia ni en la calidad de vida de los pacientes. Las falsas elevaciones debido a patologías no tumorales, pueden causar situaciones de alarma que obligan a extensos estudios que son costosos y a veces difíciles de interpretar por parte de los oncólogos y causan un malestar físico y psíquico a los pacientes.

Quizás la utilización de estos marcadores en la monitorización de la respuesta a las distintas terapias oncológicas, cuando sus valores están elevados desde el inicio y junto a otras técnicas complementarias, sea más útil como indicador pronóstico. Sin embargo, aún no se ha establecido un rol definitivo para ninguno de ellos en la práctica clínica debido a que los estudios en este campo son relativamente pequeños, retrospectivos y con poblaciones heterogéneas con respecto a factores pronósticos y/o tratamientos recibido. 


\section{Bibliografía}

1. Jiménez Lacave A, Allende Monclús M. Falsos negativos y positivos de los marcadores tumorales: sus limitaciones en la práctica clínica. Aplicaciones clínicas al CA 12-5. Rev Clin Esp 2001; 201:715-7.

2. Aziz DC, Rittenhouse HJ, Ranken R. Use and interpretation of tests in oncology. Santa Mónica (USA): Specialty Laboratories Inc, 1991.

3. Miralles C, Orea M, España P, et al. Cancer antigen CA $12-5$ associated with multiple benign and malignant pathologies. Ann Surgical Oncol 2003; 10(2):150-4.

4. Rustin GJS, Gennings JN, Nelstrop AE, et al. Use of CA12.5 to predict survival of patients with ovarian cancer. J Clin Oncol 1989; 7:1667-71.

5. Van de Burg MEL, Lammes FB, Verwij J. The role of CA 12.5 in the early diagnosis of progressive disease in ovarian cancer. Ann Oncol 1990; 1:301-2.

6. Colomer R, Ruibal A, Genolla J, Salvador L. Circulating CA 15-3 antigen in non-mammary malignancies. Br J Cancer 1989; 59:283-6.

7. Hashimoto T, Matsubara F. Changes in the tumor marker concentration in female patients with hyper-, eu-, and hypothyroidism. Endocrinol Jpn 1989; 36:873-9.

8. Bast RC, Ravdin P, Hayes DF, et al. 2000 Update of recommendations for the use of tumor markers in breast and colorectal cancer: clinical practice guidelines of the American Society of Clinical Oncology. J Clin Oncol 2001; 19(6): 1865-78.

9. Bast RC. Status of tumor markers in ovarian cancer screening. J Clin Oncol 2003; 21(10 Supplement): 200s-205s.

10. Tumor markers in gynaecological cancers-EGTM recommendations (editorial). Anticancer Res 1999; 19: 280710. 